\title{
Agresiones protésicas a la mucosa bucal en pacientes geriátricos rehabilitados con prótesis estomatológicas removibles.
}

\author{
Protesicases aggressions to the buccal mucous membrane in the geriatric \\ patients rehabilitated with removables dental prosthesis.
}

Suleydis Espasandín González. 1, Yusmel González Díaz. ${ }^{2}$, Vicia Olga Reyes Suárez. ${ }^{3}$ \& Barbara Yushimitt González Casañas. ${ }^{4}$

\section{DOI: https://doi.org/10.33262/anatomiadigital.v4i4.1900}

\begin{abstract}
.
Resumen.

The injuries to the buccal mucous Las lesiones a la mucosa bucal en los membrane in the geriatric patients constitute a problem of buccal health between the rehabilitated with removables dental prosthesis. With the objective of determinate the clinical forms of the protesicases aggressions to the buccal mucous membrane, your localization and irregularity associated as pacientes geritricos constituyen un problema de salud bucal entre los rehabilitados con protesis estomatologicas removibles. Con el objetivo de determinar las formas clínicas agudas y crónicas de las agresiones protésicas a la mucosa bucal, su localización e irregularidad protésica

${ }^{1}$ Especialista de I Grado en Estomatología General Integral y de II Grado en Prótesis Estomatológica. Máster en Urgencias Estomatológicas. Investigador Agregado. Asistente. Clínica Estomatológica Docente «Andrés Ortiz Junco». Güines, Cuba. Correo electrónico: sespasandin @infomed.sld.cu. https://orcid.org/0000-0001-9885-0342

${ }^{2}$ Especialista de I Grado en Estomatología General Integral. Clínica Estomatológica Docente «Andrés Ortiz Junco». Güines, Cuba. Correo electrónico: yusmel.diaz @ nauta.cu,

${ }^{3}$ Especialista de II Grado en Ortodoncia. Máster en Medicina Bioenergética y Natural. Investigador Auxiliar Profesora Auxiliar y Consultante. Facultad de Ciencias Médicas Mayabeque. Güines, Cuba. Correo electrónico: olga.reyes@infomed.sld.cu, (i) https://orcid.org/0000-00003-4447-6943.

${ }^{4}$ Lic en enfermería. Master en Educación Superior .Investigadora agregada. Profesora auxiliar. Facultad de Ciencias Médicas Mayabeque. Güines, Cuba. Correo electrónico: barbiy.gonzalez@ nauta.cu
\end{abstract}


well as identifying protesicoses inadequate and noxious habits in these patients an observational, descriptive and transversal study was carried out in the dental clinical "Andres Ortiz Junco", of the Guines municipality Mayabeque from October 2019 to October 2021. The universe 360 patient and the sample 80 selected for a sampling not probabilistico, deliberate and opportunistic. Signed two similar groups numerically, one with acute injuries and other with injuries cronicases. The variables in study went: form acute and cronicases of the protesica aggressions to the mucous membrane, localization areas, protesica irregularity and inadequated and noxious protesicoses habits. Carried out a cross-examination, the buccal examination and of the prosthesis in use. The statistical analysis carried out through the docima X2 y Duncan. As a result it are obtained that the $38,75 \%$ of the diagnosed injuries were subprotesises stomatitis and a 28,75\% were traumatic ulcers. The $37,40 \%$ with affectation of the palate. The 39,02 5 of the protesises it had as protesica irregularity the compresion evelope. The 48,75 of the patients were accustomed the protesises in na endless way, the 41,25 $\%$ with a faulty hygiene and a 53,75\% were smoking. Of which it is concluded that the chronic more frequente lesion went the suprotesises stomatitis and of the acute the traumatic ulcer,the more affected are the plate.

Keywords: removables dental prosthesis, geriatric patients, injuries, buccal mucous membrane, protesicases aggressions. asociada, así como identificar los hábitos protésicos inadecuados y nocivos en estos pacientes se realizó una investigacion observacional,descriptiva y transversal en la Cliníca estomatológica ". Andrés Ortíz Junco“ del municipio Guines ,Mayabeque, en el período de octubre del 2019 a octubre del 2021. El universo 360 pacientes y la muestra 80 seleccionados por un muestreo no probabilístico,intencionado y oportunista.Se formaron dos grupos iguales numéricamente, uno con lesiones agudas y otro con lesiones crónicas. Las variabes en estudio fueron: formas clínicas agudas y crónicas de las agresiones a la mucosa bucal, zonas de localización, irregularidad protésica y hábitos protésicos inadecuados y nocivos. Se realizo un interrogatorio el examen bucal y de las protesis en uso.El análisis estadístico se realizó a través de la docima $\mathrm{X} 2$ y Duncan. Como resultados se obtuvieron que el 38,75\% de las lesiones eran estomatitis subprótesis y un 28,75\% úlceras traumáticas. El $37,40 \%$ con afectación del paladar. El 39,02 \% de las prótesis tenían como irregularidad protésica la sobre compresión. El 48,75\% de los pacientes usaban la prótesis de forma continua, el $41,25 \%$ con una higiene deficiente y un $53,75 \%$ eran fumadores. De lo que se concluyó que la lesión cronica más frecuente fue la estomatitis subprótesis y de las agudas la úlcera traumatica, el áreas más afectada el paladar.La irregularidad protésica más común fue la sobre compresión. Predominó el uso continuo de la prótesis, la higiene deficiente y el tabaquismo.

$\begin{array}{llr}\text { Palabras } & \text { claves: } & \text { Prótesis } \\ \text { estomatológicas } & \text { removibles, } & \text { pacientes }\end{array}$


geriatricos, lesiones, mucosa bucal, agresiones protésicas.

\section{Introducción.}

El diseño, fabricación y aplicación de cualquier prótesis no solamente tiene como objetivo básico reponer piezas dentales y tejidos subyacentes perdidos para una mejor estética y funcionamiento, sino también para mejorar los tejidos permanentes mediante una concepción bien clara de los estados de reposo y actividad de la estructura dental con precaución de no ocasionar daños a dichos tejidos. (León 2019)

Las prótesis mal confeccionadas, o inadecuadamente conservadas que hayan perdido inevitablemente sus cualidades por continuar la persona usándolas más allá de su tiempo útil han contribuido a la aparición de lesiones malignas y premalignas en los tejidos bucales con una mayor incidencia en la población adulta, con un promedio de edad de 60 años , a pesar de las modernas técnicas clínicas y de laboratorio que aporta el desarrollo tecnológico en el área estomatológica para la fabricación de prótesis, siendo la mucosa bucal el tejido más frecuentemente afectado en asociación con el uso de las mismas (Nápoles 2016).

La mucosa bucal tiene un número ilimitado de respuestas patológicas. Éstas se conocen como lesiones elementales y son capaces de caracterizar a las enfermedades mucocutáneas, produciéndose como consecuencia de agresiones por agentes externos, o bien, son la manifestación de una enfermedad sistémica. Pueden ser primarias o secundarias. Las primarias son aquellas que se asientan sobre una mucosa previamente sana. Cuando se modifican, ya sea espontáneamente o por acción de distintos agentes, dan origen a las lesiones secundarias (Reinarte 2017).

La presencia de lesiones de la mucosa bucal afecta el estado de salud general, de las personas. Esto se debe a que son patologías acumulativas o progresivas que propician trastornos fisiológicos muy complejos que pueden llegar a afectar el estilo de comer, la forma de comunicarse, el aspecto, en ocasiones, producen dolor y molestias. El área más frecuente para el desarrollo de las lesiones es la zona que mantiene el contacto constante con la parte interna de la prótesis (Nodarse 2017).

Estas lesiones paraprotésicas pueden diagnosticarse de dos tipos: agudas y crónicas. La primera suele ser producto del uso de prótesis nuevas mal ajustadas que ejercen una presión enorme contra los tejidos blandos lo que favorece la aparición de ulceraciones o isquemia arterial y/o dolor. La segunda, son el resultado de prótesis inestables que producen roces sobre el tejido los que estimulan modificaciones graduales del tejido de sostén. Sin duda, la opción de cubrir las zonas de soporte con prótesis representa un ataque mecánico no fisiológico para los tejidos que pueden ser víctimas de irritación tisular, reacciones inflamatorias (Corona 2017). 
Debido a la elevación de la incidencia y prevalencia de lesiones a la mucosa del complejo bucal en la población geriátrica rehabilitada con prótesis estomatológica removible, actualmente se reconoce como un problema que afecta la salud bucal así como la calidad de vida de estos pacientes por lo que se hace necesario realizar la presente investigación con el objetivo de determinar las formas clínicas agudas y cónicas de las agresiones protésicas a la mucosa bucal, su localización e irregularidad protésica asociada así como identificar los hábitos protésicos inadecuados inherentes a la conducta de los portadores y los hábitos nocivos a la mucosa bucal con mayor frecuencia en estos pacientes. Para indagar el papel que juegan las prótesis estomatológicas removibles en la génesis de agresiones a la mucosa bucal al comportarse como factor de riesgo en los pacientes geriátricos y de este modo contribuir a la sensibilización de pacientes y profesionales acerca de la necesidad del control de los principales factores de riesgos protésicos y los estilos de vida saludables así como de la identificación de los grupos poblacionales más susceptibles con vistas a realizar un diagnóstico precoz y oportuno de estas lesiones.

\section{Metodologia.}

Para lograr los objetivos planteados se realizó una investigación observacional, descriptiva, de corte transversal en pacientes geriátricos rehabilitados con prótesis estomatológicas removibles tratados en la clínica estomatológica docente "Andrés Ortiz Junco“ del municipio de Güines, provincia de Mayabeque, durante el período de tiempo comprendido de octubre del 2019 a marzo del 2021.

El universo estuvo integrado por 360 pacientes geriátricos rehabilitados con prótesis estomatológicas removibles en el periodo en estudio pertenecientes a dicha área de salud y que fueron diagnosticados con algún tipo de agresión protésica a la mucosa bucal. A través de un muestreo no probabilístico, intencionado, y oportunista, se obtuvo una muestra de 80 pacientes.

Se excluyeron del estudio a los pacientes portadores de prótesis parcial fija, aquellos con lesiones bucales no portadores de prótesis y los que presentaron algún tipo de enfermedades sistémicas o tratamientos medicamentosos que puedan afectan la mucosa del complejo bucal.

Los pacientes seleccionados se dividieron en dos grupos con igualdad numérica, uno conformado por los pacientes con lesiones agudas a la mucosa bucal y el otro con lesiones crónicas de manera tal que ambos tuvieran la misma probabilidad de formar parte de la exploración previo consentimiento para participar en la investigación.

Posteriormente se realizó una exploración clínica utilizando un kit de clasificación facilitado por el departamento con el objetivo de examinar la cavidad bucala través de la observación, encaminado a la exploración de la mucosa bucal. El examen lo realizo la protésista autora de la investigación en el sillón dental auxiliándose de la luz artificial proporcionada por la lámpara del conjunto dental y mediante visión directa.

Se observó y se palpó la parte interna del labio superior y luego el inferior, conjuntamente con los rebordes alveolares correspondientes, seguidamente los carrillos y los fondos de 
los surcos, se comenzó siempre por el lado derecho. A continuación se examinó el paladar duro y blando, parte dorsal y ventral de la lengua y el suelo de la boca.

Las lesiones a la mucosa bucal se diagnosticaron según sus características clínicas en:

- Estado inflamatorio congestivo: cuando se observó en la mucosa enrojecimiento por zonas o de toda la superficie cubierta por la base protética que se acompañó por dolor a la palpación, ardor y escozor.

- Úlcera Traumática: cuando se observó solución de continuidad de un tejido con pérdida de sustancia o necrosis del mismo de color blanco grisáceo, con bordes indurados o irregulares y circunscritos por un área eritematosa.

- Hiperplasia papilar o por cámara de succión: cuando se observó aumento de tamaño de un tejido con límites definidos que correspondió con los de la cámara de vacío realizada en la prótesis o glóbulos semiesféricos enrojecidos, acumulados en el centro del paladar que se extendieron como sucesión de nódulos de 2 á $3 \mathrm{~mm}$ de diámetro, separados por fisuras de 2 a $3 \mathrm{~mm}$ de profundidad dándole aspecto verrugoso.

- Épulis fisurado: cuando se observó una masa de tejido blanda, alargada, suelta y de consistencia fibrosa, que cabalgo sobre el reborde, en ocasiones únicas, otras veces lobulada.

- Estomatitis subprotesis: cuando se observó cambios inflamatorios intrabucales restringidos a la mucosa cubierta por una prótesis removible en forma de puntos hiperérmicos aislados, de un eritema difuso que dibujo el contorno de la prótesis o que se acompañó de proyecciones papilares o digitiformes que dieron un aspecto verrugoso a la superficie del paladar.

Estas lesiones se clasificaron además en agudas y crónicas según el tiempo de evolución de las mimas. Se realizó también el examen del aparato protésico para diagnosticar los detalles relacionados con las características del estudio, tales como: presencia de alguna irregularidad protésica e higiene de la misma .Durante el examen clínico se determinó la higiene bucal de cada paciente examinado que formo parte del estudio. Se consideró como higiene bucal y de la prótesis deficiente cuando existían restos duros o blandos en las mucosas y/o superficies protésicas.

Los pacientes se interrogaron además sobre posibles hábitos protésicos inadecuados tales como uso continuo de las mismas, uso de sustancias o productos químicos para cepillarlas así como el empleo de procederes inadecuados ante la roturas de ellas. Y por último se indago sobre la práctica de hábitos nocivos como el tabaquismo, el alcoholismo y el consumo de dieta inadecuada.

Se operacionalizaron como variables de interés: las formas clínicas agudas y crónicas de las agresiones a la mucosa bucal, zonas de localización de las lesiones, irregularidad protésica asociada a la agresión, hábitos protésicos inadecuados y nocivos.

Todos estos datos fueron recogidos en una encuesta confeccionada al efecto para el posterior procesamiento de la información y los resultados se presentaron en tablas 
utilizando el porcentaje como unidad resumen. Se analizó cada tabla, y se realizaron comparaciones con los hallazgos de otros autores sobre la base de un análisis inductivo y deductivo que permitió dar salida a los objetivos propuestos, y así se emitieron conclusiones y recomendaciones.

La información recogida en la encuesta fue procesada mediante la creación de una base de datos en formato electrónico, Microsoft Access. Para el análisis de los datos se emplearon técnicas de estadísticas descriptivas y se calculó la distribución de frecuencias absolutas y relativas.

El análisis estadístico de los porcentajes se realizó, mediante tablas de contingencia para las diferentes variables en estudio a través de la dócima $X^{2}$ (Steel and Torrie 1988). En las tablas donde se combinaron dos factores se determinó la interacción entre los mismos. En los casos en que se alcanzaron diferencias significativas $(\mathrm{P}<0.05, \mathrm{P}<0.01$ ó $\mathrm{P}<0.001)$ se aplicó la dócima de comparación Múltiple de Duncan (1955). Para el procesamiento de la información se utilizó el software estadístico InfoStat2012 y ComparPro (análisis de Duncan), la edición del trabajo se realizó con el uso de Microsoft Excel y Word.

Para llevar a efecto la investigación se tuvieron en cuenta las siguientes consideraciones éticas relacionadas con los datos pertenecientes a las personas objeto de estudio: a todos los pacientes antes de iniciar la investigación se les informó en qué consistía el estudio y se les pidió por escrito su aprobación para participar en la investigación mediante el consentimiento informado. Además, se contó con la aprobación de la Dirección de la Clínica estomatológica donde se ejecutó la investigación, así como del Comité de ética médica del Municipio para ejecutar el estudio.

\section{Resultados}

En la "tabla 1", al analizar la distribución de lesiones a la mucosa bucal según su forma clínica de presentación en los pacientes geriátricos examinados, se observan las cifras más elevadas en la estomatitis subprotesis entre las lesiones crónicas con un 38,75\% con una elevada significación estadística respecto a los restantes tipos de lesiones crónicas y agudas estudiadas. Las úlceras traumáticas $(28,75 \%)$ y los estados inflamatorios agudos congestivos $(21,15 \%)$ le siguieron en orden de aparición con un comportamiento estadístico similar entre ellas. Los demás estados crónicos mostraron porcentajes muy bajos como la hiperplasia papilar o por cámara de succión con tan solo un 1,25\%.

Tabla 1. Formas clínicas agudas y crónicas de las lesiones bucales en los pacientes con agresiones protésicas a la mucosa bucal. Güines .2021

\begin{tabular}{lccc}
\hline \multicolumn{2}{c}{ Formas clínicas de las lesiones bucales } & \multicolumn{2}{c}{ Cantidad de pacientes } \\
\cline { 2 - 4 } & Agudas & \multicolumn{2}{c}{ No. } \\
\hline & & 17 & $21.25 \mathrm{~b}$ \\
\hline Estado inflamatorio congestivo & 23 & $28.75 \mathrm{~b}$ \\
Úlcera traumática & 40 & 50 \\
Subtotal & Crónicas & 1 & $1.25 \mathrm{~d}$ \\
\hline \multicolumn{2}{l}{ Hiperplasia papilar o por cámara de succión } & 1 & \\
\hline
\end{tabular}


Tabla 1. Formas clínicas agudas y crónicas de las lesiones bucales en los pacientes con agresiones protésicas a la mucosa bucal. Güines .2021 (continuación)

\begin{tabular}{|c|c|c|}
\hline \multirow[t]{2}{*}{ Formas clínicas de las lesiones bucales } & \multicolumn{2}{|c|}{ Cantidad de pacientes } \\
\hline & No. & $\%$ \\
\hline \multicolumn{3}{|l|}{ Crónicas } \\
\hline Épulis Fisurado & 8 & $10.00 \mathrm{c}$ \\
\hline Estomatitis subprotesis & 31 & $38.75 a$ \\
\hline Subtotal & 40 & 50 \\
\hline Total & 80 & 100 \\
\hline EE y Sign & \multicolumn{2}{|c|}{ $\pm 4.47^{* * *}$} \\
\hline
\end{tabular}

EE: Error estándar

Sign: Significación estadística $\quad * * * \mathrm{P}<0,001$

a, b Superíndices diferentes indican diferencias significativas según Duncan (1955)

La tabla 2 muestra las zonas de localización de lesiones bucales diagnosticadas en los pacientes objetos de estudios, donde la localización que reúne las mayores cifras resulto ser las del paladar para un $37,40 \%$ y la del reborde alveolar con un $31,71 \%$ con igual comportamiento estadístico entre ellas y con diferencias de elevadas significación respecto al resto de las regiones Le siguen en orden de aparición y con similar comportamiento las lesiones ubicadas en el fondo del surco y en los carrillos para un $12,20 \%$ y un $6,50 \%$ respectivamente las que difieren estadísticamente respecto a las lesiones ubicadas en los frenillos, exostosis óseas, línea oblicua, suelo de boca, labio y lengua que también mostraron entre ellas un comportamiento estadístico similar y con los más bajos porcentajes.

Tabla 2. Zonas de localización de las lesiones en los pacientes geriátricos con agresiones protésicas a la mucosa bucal. Güines. 2021

\begin{tabular}{lcc}
\hline \multicolumn{1}{c}{ Zonas de localización } & No. & $\%$ \\
\hline Labio & 2 & $1,63 \mathrm{c}$ \\
Carrillo & 8 & $6,50 \mathrm{~b}$ \\
Paladar & 46 & $37,40 \mathrm{a}$ \\
Lengua & 1 & $0,81 \mathrm{c}$ \\
Suelo de boca & 2 & $1,63 \mathrm{c}$ \\
Frenillos & 5 & $4,07 \mathrm{c}$ \\
Reborde alveolar & 39 & $31,71 \mathrm{a}$ \\
Línea oblicua & 2 & $1,63 \mathrm{c}$ \\
Fondo de surco & 15 & $12,20 \mathrm{~b}$ \\
Exóstosis óseas & 3 & $2,44 \mathrm{c}$ \\
\hline & &
\end{tabular}

EE: Error estándar

Sign: Significación estadística $\quad * * * \mathrm{P}<0,001$

a, b Superíndices diferentes indican diferencias significativas según Duncan (1955) 
La tabla 3 muestra que las cifras más elevadas de irregularidades protésicas asociadas a las agresiones a la mucosa bucal en los pacientes geriátricos examinados le corresponden a la sobre compresión $(39,02 \%)$ respecto a los restantes tipos de irregularidades protésicas con diferencia de gran significación estadística. Los puntos de contacto prematuros le siguen en orden decreciente con un $24,39 \%$ precedido de los bordes sobreextendidos con el 11,38\%, la ausencia de resalte y de alivio con el $8,94 \%$ en cada uno de ellos, estas tres últimas mostrando un comportamiento estadístico similar entre ellas. El resto de las irregularidades estudiadas se presentaron en muy bajos porcientos y con un comportamiento similar.

Al relacionar los diferentes tipos de irregularidad protésica con la localización de la lesiones a la mucosa bucal no se encontró significación estadística en las lesiones al labio, la lengua, el suelo de boca, línea oblicua y en las exostosis óseas mientras que las restantes localizaciones si mostraron diferencias de una elevada significación estadística. La sobre compresión irregularidad protésica más representativa se presentó en un 21,14\% de los casos en la cima de los reborde alveolares residuales y en un 16,26 \% en el paladar. Otra irregularidad causante de lesiones al paladar fueron los puntos de contactos prematuros que también produjeron afectaciones notables (14.63\%).Las lesiones en el fondo surco se presentaron mayormente por bordes sobre extendidos con un 9,76\% y 2,44\% respectivamente mientras que las lesiones en el carrillo estuvieron asociadas a la ausencias de resalte posterior con un $6,50 \%$ y en los frenillos por la inexistencias de escotaduras para un $4,06 \%$.

Tabla 3. Irregularidades protésicas asociadas de las agresiones a la mucosa bucal en los pacientes geriátricos según localización de la lesión

\begin{tabular}{|c|c|c|c|c|c|c|c|c|c|c|c|c|c|c|c|c|c|}
\hline \multirow{3}{*}{$\begin{array}{l}\text { Localización } \\
\text { de la lesión }\end{array}$} & \multicolumn{17}{|c|}{ Irregularidades protesicas } \\
\hline & \multicolumn{2}{|c|}{$\begin{array}{l}\text { Nódulos } \\
\text { de acrilico }\end{array}$} & \multicolumn{2}{|c|}{$\begin{array}{l}\text { Bordes } \\
\text { filosos }\end{array}$} & \multicolumn{2}{|c|}{$\begin{array}{l}\text { Bordes } \\
\text { sobrextendit- } \\
\text { dos }\end{array}$} & \multicolumn{2}{|c|}{$\begin{array}{l}\text { Ausencia } \\
\text { de resalte. }\end{array}$} & \multicolumn{2}{|c|}{$\begin{array}{l}\text { Sobte } \\
\text { compresión }\end{array}$} & \multicolumn{2}{|c|}{$\begin{array}{c}\text { Inexistencia } \\
\text { de } \\
\text { encotaduras }\end{array}$} & \multicolumn{2}{|c|}{$\begin{array}{l}\text { Ausencia } \\
\text { de alivio }\end{array}$} & \multicolumn{2}{|c|}{$\begin{array}{l}\text { Puntos de } \\
\text { contacto }\end{array}$} & \multirow[t]{2}{*}{$\begin{array}{l}\text { EEy } \\
\text { Sign }\end{array}$} \\
\hline & No & 6 & No. & $\%$ & No. & $\%$ & No & $\%$ & No & $\%$ & No & 96 & $\mathrm{No}$ & $\%$ & No & $\%$ & \\
\hline Labio & - & - & - & - & - & - & 2 & 1.6 & - & - & - & - & - & - & - & - & $\begin{array}{c}=0.40 \\
\text { NS }\end{array}$ \\
\hline Carrillo & - & - & - & - & - & - & 8 & $\begin{array}{c}6.50 \\
\mathbf{a}\end{array}$ & - & - & - & - & - & - & - & - & $=0.81^{\cdots \cdots}$ \\
\hline Paladar & - & $=$ & - & + & - & - & - & - & 20 & $\begin{array}{c}16.26 \\
\mathrm{a}\end{array}$ & $=$ & - & 8. & $\begin{array}{c}6.50 \\
b\end{array}$ & 18 & $14.63 a$ & $=1.90 \cdots$ \\
\hline Lengua & - & - & - & - & - & - & 1 & 0.81 & 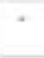 & - & - & - & - & - & - & - & $\begin{array}{c}10.29 \\
\text { NS }\end{array}$ \\
\hline $\begin{array}{l}\text { Suelo de } \\
\text { boca }\end{array}$ & - & - & - & - & 2 & 1.63 & - & - & - & - & - & - & - & - & - & - & $\begin{array}{c} \pm 0.41 \\
\text { NS }\end{array}$ \\
\hline Frenillos & - & - & - & - & - & - & - & - & + & . & 5 & $4.06 a$ & - & - & - & - & $=0.64 \cdots$ \\
\hline $\begin{array}{l}\text { Reborde } \\
\text { alveolar }\end{array}$ & 1 & $0.81 e$ & - & - & - & - & - & - & 26 & $\underset{a}{21.14}$ & - & - & - & - & 12 & $9.76 b$ & $\begin{array}{l}=1.76 \\
* \approx 0\end{array}$ \\
\hline Linea oblicua & - & - & - & - & - & - & - & - & 1 & 0.813 & - & - & 1 & $\begin{array}{l}0.81 \\
301\end{array}$ & - & - & $=0.41 \mathrm{NS}$ \\
\hline $\begin{array}{l}\text { Fondo de } \\
\text { surco }\end{array}$ & - & - & 3 & $2.44 b$ & 12 & $9.76 \mathrm{a}$ & - & - & - & - & - & - & - & 0.06 & - & - & $=1.10 \cdots$ \\
\hline $\begin{array}{l}\text { Exóstosis } \\
\text { ósea }\end{array}$ & - & - & - & - & - & - & - & - & 1 & 0.813 & - & - & 2 & $\begin{array}{l}1.62 \\
602\end{array}$ & - & - & $=0.50 \mathrm{NS}$ \\
\hline Total & 1 & $0.81 \mathrm{~d}$ & 3 & $2.44 d$ & 14 & $1138 \mathrm{c}$ & 11 & $\begin{array}{c}8.94 \\
c\end{array}$ & 48 & $\begin{array}{c}39.02 \\
a\end{array}$ & 5 & $4.06 \mathrm{~d}$ & 11 & $\begin{array}{c}8.94 \\
c\end{array}$ & 30 & $24.39 \mathrm{~b}$ & $=2.98 \cdots$ \\
\hline
\end{tabular}

EE: Error estándar

Sign: Significación estadística

$* * * \mathrm{P}<0.001 \quad \mathrm{NSP}>0.05$ 
En la tabla 4 al analizar los hábitos protésicos inadecuados y nocivos a la mucosa dentro de la población objeto de estudio se observa un mayor número de pacientes con diagnóstico de lesiones bucales entre aquellos pacientes que usan las prótesis de forma continua y que poseen una higiene bucal y de la prótesis deficiente para un 48,75\% y un $41,25 \%$ respectivamente comportándose de forma similar entre ellas y con diferencias altamente significativas respecto a los pacientes que actúan de forma inadecuada ante la rotura de la prótesis $(7,50 \%)$ o de aquellos que hacen uso de sustancias o productos químicos para cepillar sus prótesis $(2,50 \%)$ que mostraron bajos porcentajes y diferente comportamiento entre ellas.

Las lesiones bucales también fueron más numerosas entre los fumadores con un 53,75\% cifras que difieren con gran significación estadística del resto de los hábitos nocivos, presentándose con bajos porcentajes los que consumen bebidas alcohólicas diariamente $(8,75 \%)$, aunque no despreciable resultó ser el 37,5\% de pacientes que mantenían una dieta inadecuada.

Tabla 4. Hábitos protésicos inadecuados y nocivos en los pacientes geriátricos con lesiones a la mucosa bucal

\begin{tabular}{lll}
\hline Hábitos & \multicolumn{2}{l}{ Cantidad de pacientes } \\
\cline { 2 - 3 } & No. & $\%$ \\
\hline Protésicos inadecuados & 39 & $48.75 \mathrm{a}$ \\
Uso continuo de la prótesis & 33 & $41.25 \mathrm{a}$ \\
Higiene bucal y de la prótesis deficiente & $2.50 \mathrm{c}$ \\
Uso de sustancias o productos químicos para cepillar las & 2 & $7.50 \mathrm{~b}$ \\
prótesis & 6 & \\
Proceder inadecuado ante la rotura de la prótesis & & 100 \\
EE y Sign & $\pm 4.80^{* * *}$ & \\
Total & 80 & $53.75 \mathrm{a}$ \\
Nocivos a la mucosa & & $8.75 \mathrm{~b}$ \\
Tabaquismo & 43 & $37.5 \mathrm{~b}$ \\
Alcoholismo & 7 & \\
Dieta inadecuada & 30 & 100 \\
EE y Sign & $\pm 5.27^{* * *}$ & \\
Total & 80 & \\
\hline EE Error & &
\end{tabular}

EE: Error estándar

Sign: Significación estadística $\quad * * * \mathrm{P}<0.001$

a, b Superíndices diferentes indican diferencias significativas según Duncan (1955)

Aunque las prótesis dentales son elementos artificiales que se usan con la finalidad de devolverle al paciente sus dientes y garantizar las funciones del sistema estomatognático, esta acción de cubrir las zonas de soporte, constituye una agresión mecánica no fisiológica para el tejido, que puede causar acciones irritantes sobre esta, romper el equilibrio adaptativo y producir, en consecuencia, una irritación tisular, con sus consiguientes reacciones inflamatorias, hasta producir lesiones de la cavidad, un problema importante de salud. 
Con esta investigación se corroboró que la estomatitis subprótesis es la lesión más prevalente en la mucosa bucal de los pacientes portadores de prótesis acrílicas removibles mucosoportadas.

En estudios realizados se evidencia la relación que existe entre la aparición de lesiones bucales y el uso de prótesis estomatológicas semejante a lo hallado en el presente estudio donde un número considerable de pacientes fueron diagnosticados con algún tipo de lesión en los tejidos de soporte o adyacentes a las prótesis desde un periodo de escasos dos años post instalación hasta varios años de uso (Corona 2017, Díaz 2017 y Vázquez 2019).

De ahí la necesidad de poner énfasis en la necesidad de su cambio cada cinco años y el chequeo periódico en estos pacientes en correspondencia con las variaciones fisiológicas propias de la edad.

En un estudio realizado en Cienfuegos sobre lesiones a la mucosa bucal asociadas al uso de prótesis la lesión más observada fue la estomatitis subprótesis con un 83,33 \% relacionada con largos periodos de tiempo de exposición a la prótesis, resultado que coinciden con los de esta investigación (Vázquez 2019).

También concuerda con otra investigación realizada en pacientes edéntulos totales donde la principal lesiones de la mucosa oral asociadas al uso de la prótesis en estos pacientes fue la estomatitis protésica con un 71,43\% (León 2019).

En general la localización de las lesiones diagnosticadas en esta investigación coincide con estudios anteriores realizados donde mencionan al paladar como la región anatómica más afectada por lesiones bucales (León 2016, Nápoles 2016, Corona 2017, Díaz 2017 y Vázquez 2019).

Un estudio realizado en Brasil refleja que las lesiones del paladar con un $45.5 \%$ fueron las que más predominaron en los pacientes, producto de prótesis nuevas mal ajustadas que ejercen una presión enorme contra los tejidos blandos, así como el uso de prótesis inestables que producen roces sobre el tejido los que estimulan modificaciones graduales del tejido de sostén (Dantas 2019)

En el presente estudio el reborde alveolar tiene un nivel de afectación estadísticamente similar al paladar resultado contario al encontrado en la investigación donde las lesiones en esta región no son significativas como tampoco lo son en las restantes regiones estudiadas (Núñez 2017) tal como lo hallado en esta investigación.

Se reafirma el hecho de que la prótesis es un elemento que actúa como un cuerpo extraño provocando reacciones de los tejidos colindantes, que va traumatizando las mucosas que la soportan, y en la medida que pasa el tiempo producen daño a los tejidos apareciendo las lesiones. Además el paladar es la zona anatómica más extensa cubierta por la prótesis lo que pudiera incrementar la probabilidad de sufrir más lesiones. 
La funcionalidad de la prótesis significa salud para el paciente y mejoría de su calidad de vida por lo que es fundamental evitar que los elementos del sistema estomatognático sufran agresiones producto al uso de prótesis con irregularidades durante su construcción. De ahí que el análisis de la calidad de las prótesis resulta, por tanto, de especial importancia y responsabilidad del profesional así como del equipo de trabajo ya que el principal propósito en el servicio de prótesis es precisamente rehabilitar al paciente desdentado total o parcial desde todo punto de vista: funcional, estético, psíquico y social, lo que eleva su autoestima, favorece sus relaciones sociales y mejora su estado de salud.Una prótesis con problemas mecánicos que atentan contra la calidad de la misma es también capaz de producir lesiones en la mucosa bucal. El grado de satisfacción del portador de prótesis dental no será igual si aparecen lesiones en la mucosa bucal porque estas podrán ocasionar molestias e inadaptación a la misma que incluso podrán llevarlo a retirársela, lo cual sería un fracaso del tratamiento rehabilitador.

Es de destacar que los resultados obtenidos demuestran que existe relación entre el lugar donde aparecen las lesiones y las causas o irregularidades protésicas que la provocan. En este sentido se han presentado limitaciones para el análisis comparativo de los resultados obtenidos con los de otros investigadores debido a las escasas investigaciones a nivel mundial y nacional que estudien las variables aquí utilizadas aunque se coinciden con Espasandín, (2015) y Ramírez, (2019) quienes afirman que "la falta de estabilidad, las inadecuadas relaciones céntricas o interferencias en la oclusión, se traducen en presiones que pueden causar alteraciones, o bien alterar canales circulatorios que originan lesiones de carácter degenerativo, como la atrofia del epitelio y el debilitamiento de sus funciones de defensa y protección, lo que trae como consecuencia la aparición de lesiones a la mucosa bucal del tipo de la estomatitis subprotesis y las úlceras“ p 90 - 91, p215.

Afirmación está respaldada con los resultados aquí obtenidos donde la sobre compresión y los puntos de contactos prematuros fueron las irregularidades protésicas más comunes afectando fundamentalmente al paladar y a la cima del reborde alveolar residual y por quien al estudiar la presencia de lesiones bucales en un grupo de pacientes portadores de prótesis afirma que el 35,29 \% sufría de presión causada por la prótesis en alguna zona de la mucosa bucal en contacto directo con el aparato protésico (Díaz 2007).

Resultados similares en cuanto al número de pacientes con puntos de contactos prematuros se obtienen en un estudio anterior realizado en Camagüey sobre la influencia de la rehabilitación protésica en la calidad de vida de los pacientes portadores de prótesis donde este problema mecánico de las prótesis fue uno de los más frecuentes entre los examinados, pero con un porciento mucho menor (13.9\%) (Ugarte 2014).

Al analizar los problemas mecánicos resalta el hecho que el borde periférico debe ajustar en los tejidos movibles y tenga el diseño necesario para no interferir en sus movimientos normales, ni en la nutrición de los tejidos de soporte, este es un requisito que debe ser cumplido en la confección de las prótesis totales para así contribuir al principio biomecánico de retención de las mismas. Por tanto, se coincide con el criterio de Ugarte, (2014) de que si "los flancos de las mismas se encuentran sobre extendidos o filosos y las 
inserciones frénicas se encuentran sin liberar, constituyen factores que no solo atentan contra la retención y estabilidad del aparato protésico sino que son capaces de agredir a la mucosa bucal creando en ella alteraciones patológicas de diferente índole“ p 622.

Referente a los flancos sobreextendidos y a las inserciones frénicas sin liberar se difiere de los resultados obtenidos en un estudio donde estos dos factores fueron las principales dificultades encontradas en las prótesis de los pacientes, con $30.2 \%$ y un $18.6 \%$ respectivamente (Ley 2010).

En otro estudio revisado se encontraron resultados similares a los obtenidos en esta investigación en cuanto al uso de la prótesis de forma continua y a la higiene deficiente donde solo el 18,7 \% de los aparatos protésicos estaban higienizados y el 43,7\% de los pacientes de la muestra usaban sus prótesis durante 24 horas (Nodarse 2019)

Es válido destacar que los pacientes que usan prótesis por tiempo prolongado y no se la quitan durante las horas de sueño sufren lesiones de la mucosa bucal por causas mecánicas, debido a que la prótesis se comporta como un factor traumático local, que al estar en contacto con la mucosa más allá del tiempo recomendado al día, irrita la mucosa, se producen cambios fisiológicos y se convierten con el tiempo en lesiones del tejido blando. Debe señalarse que la higiene bucal no sólo incluye la limpieza de los dientes remanentes (en el caso de que existan), sino también la limpieza del aparato protético y el enjuagatorio de los tejidos blandos que se encuentran en contacto con la prótesis. Una higiene deficiente trae como consecuencia el depósito de placa dentobacteriana en el interior de la base protésica y los dientes, fundamentalmente en aquellas prótesis que no están bien adaptadas, lo que constituye al desarrollo de microorganismos oportunistas como la Cándida albicans, incluso pueden proliferar bacterias en forma de cocos y bacilos, a causa de la descomposición de los alimentos.

García, (2010) afirma que "el riesgo de poseer lesiones paraprotésicas aumenta cuando no se logra una buena higiene bucal de manera tal que el riesgo de que aparezca una lesión en los tejidos de soporte y adyacentes a las prótesis aumenta en más de dos veces en aquellas personas con una higiene deficiente" p $16-17$

En este estudio predominó la higiene bucal deficiente sobre la eficiente entre los portadores de prótesis con diagnóstico de algún tipo de lesión bucal semejante a los pobres índices de higiene bucal reportados por otros autores que hacen énfasis en lo poco que se conoce con respecto a la higiene de las prótesis y más aún, lo poco que se ha hecho para implantarlo en la práctica dental, lo que desencadena en los pacientes, la percepción de que la higiene bucal y de las prótesis dentales no es tan importante, desconociendo las repercusiones de la mala higiene bucal de las prótesis dentales, sobre los tejidos blandos adyacentes (Díaz 2007 y Ugarte 2014).

Las elevadas cifras de una deficiente higiene bucal y de las prótesis pudiera atribuirse a que las habilidades y destrezas de las personas con el avance de los años se pierden paulatinamente y más aún cuando comienzan los temblores de la vejez que impiden una 
minuciosa limpieza de las mismas, si se tiene en cuenta que el estudio se realiza en población geriátrica.

La correcta higiene de la prótesis debe realizarse sin el uso de productos químicos que no son los indicados para este fin: como detergentes, jabón, cloro o cualquier otro desinfectante; pues estos provocan deterioro de los materiales de construcción de la prótesis haciéndolos más vulnerables y favoreciendo la consiguiente acumulación de cálculo y placa dentobacteriana. Debido al abuso de estas sustancias o métodos de limpieza inadecuados se incrementan las agresiones que pueden producir a los tejidos blandos bucales al actuar estos productos químicos como irritantes serios a dichos tejidos.

La carencia significación estadística en este estudio en cuanto al uso de sustancias o productos químicos para cepillar o higienizar sus prótesis pudiera atribuirse a que dentro de las indicaciones post-instalación de las prótesis se explica por el personal profesional la frecuencia y la forma en que se deben limpiar las prótesis haciendo énfasis en usar solo agua y pasta dental y evitar el uso de productos químicos que son absorbidos por las bases acrílicas que son porosas y que luego se van expulsando poco a poco durante el uso de las prótesis que están en contacto continuo y directo con los tejidos bucales que le sirven de soporte y los tejidos vecinos como la lengua, labios y carrillos.

En una investigación realizada en la Facultad de Odontología de Córdoba, encuestaron a setenta personas portadoras de prótesis sobre aspectos relacionados con la higiene de sus prótesis, y el $63 \%$ manifestó no saber cómo higienizarlas y referente al uso de sustancias para la limpieza de las mismas el $80 \%$ de los entrevistados lo hace con cepillo de dientes y pasta dental resultado similar al de este estudio (Oliveira 2016).

Un estudio realizado determinó el estado prostodóncico y aspectos de salud bucal, en los pacientes que solicitaron reparaciones protésicas la frecuencia del uso de métodos incorrectos de higiene bucal alcanzó altos porcentajes con el 95,10\% resultados estos que difieren con los aquí obtenidos (Romero 2015)

Ante cualquier daño que presente la prótesis no debe ser reparada en la casa, pues esto puede causar daño a la dentadura hasta el punto que se dificulte su ulterior reparación por parte del estomatólogo lo que imposibilita su uso e incluso crea problemas en la cavidad bucal; pues los pegamentos de uso casero contienen a menudo químicos peligrosos y no se deben usar en las prótesis, ya que, si esta no ajusta correctamente pude traer consigo irritación y úlceras, entre otras complicaciones.

En los servicios de prótesis del país se realizan actividades educativas encaminadas a informar e instruir a los portadores de prótesis en general del proceder que deben seguir con las mismas cuando estas no se usan, para garantizar su protección y evitar roturas por causa de algún accidente de índole doméstico o ante la rotura de algunas de sus partes donde lo más aconsejable es acudir al estomatólogo en busca de solución; lo que puede dar respuesta a los resultados aquí obtenidos sobre el proceder ante las roturas de las prótesis donde la mayoría de los pacientes encuestados refirieron visitar al estomatólogo ante la rotura de la prótesis. 
Estudios realizados reafirman que un gran número de portadores de prótesis ante la rotura de alguna de sus partes acuden con gran frecuencia a los servicios de prótesis estomatológicas para su reparación, similar a la conducta asumida en este estudio entre los rehabilitados protésicamente (Romero 2015, Martínez 2016 y Espasandin 2016) .

En el presente estudio no fue significativo la ingestión de una dieta inadecuada dentro de los hábitos nocivos como factor de riesgo para la aparición de lesiones bucales como es el caso de la ingestión de alimentos o bebidas calientes, como el café que producen irritación crónica de la mucosa bucal, pues estas irritan desde un primer contacto con los tejidos bucales, sin importar el modo y la cantidad de bebida que se ingiere.

Según Cruz, (2016) "la ingestión de bebidas alcohólicas puede ser también cancerígeno por diferentes mecanismos, pero su más importante ruta es su oxidación a acetaldehído, que interfiere con la síntesis y la reparación del ADN. El alcohol actúa como un solvente que favorece la exposición de la mucosa oral a los carcinógenos y la captación celular de los mismos" p135. Afortunadamente entre los entrevistados se reportó muy bajos porcientos de pacientes alcohólicos.

La alta significación estadística mostrada por el consumo del tabaco reafirma lo reportado en otro estudio realizado donde este hábito resulto ser el principal factor de riesgo asociado con la aparición de lesiones bucales y consecuentemente con el cáncer bucal por sus múltiples sustancias carcinogénicas. (Domínguez 2016).

El humo de tabaco genera carcinógenos, y los fumadores exponen su aparato aerodigestivo superior a la acción de los mismos, lo que provoca cambios en esa mucosa que persisten por años, aún si el paciente deja de fumar.

Un estudio anterior reporta que el $35 \%$ de la población encuestada, ignora el daño que puede causar el hábito de fumar para la salud bucal a pesar de las fuertes campañas educativas que en este sentido se han desarrollado en el país. (Reinarte 2017)

No obstante bebe tenerse en cuenta que Cuba produce el mejor tabaco del mundo y por tradición las generaciones se transmiten este hábito tan dañino para la salud bucal y general, lo que puede influir en los resultados obtenidos

A todo lo anterior debe añadirse que el envejecimiento supone un aumento del riesgo de la presencia de alteraciones y patologías de la mucosa bucal como consecuencia de la acumulación de factores internos fisiológicos que provocan enfermedades e inducen cambios bioquímicos, funcionales y estructurales.

Los profesionales al examinar la cavidad bucal en los adultos mayores, deberán considerar las manifestaciones bucales, alteraciones sistémicas y también lesiones ocasionadas por factores locales en el portador de prótesis estomatológicas, o por ambas causas a la vez. Se debe insistir en dedicar parte de los esfuerzos a observar cómo se comportan estas en orden de prevalencia, así como estudiar la incidencia de posibles factores de riesgo en su aparición, analizándolos, interrelacionándolos en conjunto como parte del mecanismo mediante el cual se producen estas entidades. 


\section{Conclusiones}

- En los pacientes con agresiones protésicas a la mucosa bucal predominó la estomatitis subprótesis dentro de las lesiones crónicas con gran significación estadística y entre las lesiones agudas las úlceras traumáticas, destacándose el paladar como la zona más afectada.

- Las irregularidades protésicas más frecuentes asociadas a las agresiones a la mucosa bucal corresponden a la sobre compresión

- Dentro de los hábitos protésicos inadecuados predominó el uso continuo de la prótesis y la higiene deficiente además del tabaquismo como hábito nocivo a la mucosa bucal.

\section{Referencias bibliográficas.}

Corona Carpio MH, Ramón Jimenez R, Urgellés Matos W. (2017). Lesiones de la mucosa bucal en adultos mayores con prótesis dentales totales. MEDISAN, 21(7):813818.http://scielo.sld.cu/scielo.php?script=sci_arttext\&pid=S10293019 2017000700006\&lng=es

Dantas de Medeiros, Fabianna; De Araújo-Silva, Thiago; Alves Ferreira, Kleiton; Oliveira-Moura, Jamile; Cavalcanti-Lima, Isabela; Guerra-Seabra E, (2015). Uso de prótesedentária e suarelaçãocomlesõesbucais. Rev salud pública https://revistas.unal.edu.co/index.php/revsaludpublica/article/view/34322

Díaz Y, Martell IC, Zamora JD. (2017). Afecciones de la mucosa oral encontradas en pacientes geriátricos portadores de prótesis estomatológicas. RevCubana Estomatología, 44 (3). http://bvs.sld.cu/revistas/est/vol44_3_07/est0230 7.html

Díaz Martell Y, Martell Forte I de la C, Zamora Díaz Javier D, (2007). Afecciones de la mucosa oral encontradas en pacientes geriátricos portadores de prótesis estomatológicas. Rev Cubana Estomatol, 44(3). http://scielo.sld.cu/scielo.php?script=sci_arttext\&pid=S00347507200700030000 $2 \& \operatorname{lng}=$ es

Domínguez Santana L, Valdés Herrera M, Cabrera Cabrera D, Rodríguez Pérez L. (2016). Estado de salud bucal de la población geriátrica de un consultorio del Policlínico Rampa. Revhabanciencméd; 22(1). http://revcmhabana.sld.cu/index.php/rcmh

Espasandín González S, Reyes Suárez V, Soler Gil M, Pérez Acosta K. (2015) Factores de riesgo asociados a la aparición de la estomatitis subprótesis. Revista de Ciencias Médicas La Habana; http://revcmhabana.sld.cu/index.php/rcmh/article/view/70910

Espasandín González S, Martínez Borges A, Reyes Suárez VO, Pérez Acosta K, (2016). Frecuencia de las urgencias en el servicio de prótesis. Revista de Ciencias Médicas de La Habana 22(1). http://revcmhabana.sld.cu/index.php/rcmh/article/view/929 
García Alpizar B, Capote Valladares M, Morales Montes de Oca.Benet TJ. (2012) Prótesis totales y lesiones bucales en el adulto mayores Institucionalizados. Revista Finlay; 2(1).

León-Velastegui M.A, Gavilanes-Bayas N.A, Mejía-Navas E.X. (2019). Lesiones de la mucosa oral asociadas al uso de prótesis odontológicas en pacientes edéntulos totales. Dominio de las Ciencias. https://dominiodelasciencias.com/ojs/index.php/es/rt/printerFriendly/880/html

Ley Sifontes L, Silva Martínez Y, Puig Capote E, Jesús Nápoles I de, Díaz Gómez SM,(2013). Comportamiento de la estomatitis subprótesis. AMC; 14(1). http://scielo.sld.cu/scielo.php?script=sci_arttext\&pid=S10250255201000010000 $9 \& \operatorname{lng}=$ es\&nrm=iso\&tlng=es

Martínez Borges A.C, González S.E, González Vivero S.J, Rivero Villalón M, (2016). Urgencias en el servicio de prótesis estomatológica. REVISTA DE CIENCIAS MÉDICAS. https://www.medigraphic.com/pdfs/revciemedhab/cmh-2016/cmh162d.pdf

Miguel Cruz Pedro Antonio, Niño Peña Aluett, Batista Marrero Kelia, Miguel-Soca Pedro Enrique. Factores de riesgo de cáncer bucal. (2016). Rev Cubana Estomatol ; 53( 3 ): 128-145. Disponible en: http://scielo.sld.cu/scielo.php?script=sci_arttext\&pid=S00347507201600030000 $6 \& \operatorname{lng}=$ es.

Nápoles González I de J, Rivero Pérez O, García Nápoles CI, Pérez Sarduy D. (2016) Lesiones de la mucosa bucal asociadas al uso de prótesis totales en pacientes geriatras. Arch Med Camagüey, 20(2) http://scielo.sld.cu/scielo.php?script=sci_arttext\&pid=S10250255201600020000 $8 \& \operatorname{lng}=\mathrm{es}$

Nodarse Lazo R. (2019). La prótesis estomatológica como factor de riesgo de lesiones premalignas y malignas en la cavidad bucal. Rev. Arch Med Camagüey, V23 (4). http://revistaamc.sld.cu/

Nuñez Medrano Nervo, Calixto Ruiz Máximo, (2017). Impacto en la calidad de vida relacionada con salud bucal de la rehabilitación protésica convencional en pacientes desdentados completos, atendidos en el servicio público chileno. Revista Científica Mundo de la Investigación y el Conocimiento. V 1(4). https://www.google.com/url?sa=t\&rct=j\&q=\&esrc=s\&source=web\&cd=\&ved= 2ahUKEwipxG25cbvAhV9RjABHXu6Di8QFjADegQIDBAD\&url=https\%3A $\% 2 \mathrm{~F} \% 2 \mathrm{Fdialnet}$.unirioja.es\%2Fdescarga\%2Farticulo\%2F6732729.pdf\&usg=AO vVaw1c0RsIzvcutiYSmRXIU1iK

Oliveira, Andreza; Carvalho, Helene; Tramontani, Bruna; Sayed, Thays; Santos, Livia; Oliveira, Geraldo; Cantisano M, (2016). Prevalence of oral lesionscausedbyremovable. Rev Bras Odontol, 73(2). 
https://www.researchgate.net/profile/Picciani_BLS/publication/305216698_Prev alence_of_oral_lesions_caused_by_removable_prosthetics/links/578d078808ae5 9aa66815367/Preva lence-of-oral-lesions-caused-by-removable-prosthetics.pdf

Reinarte Villafuerte J.(2017). El bienestar y calidad de vida del adulto mayor, un reto para la acción intersectorial. Medisur, v15 http://scielo.sld.cu/scielo.php?script=sci_arttext\&pid=S1727897X20170001000 12

Ramírez Carballo MM, Gómez Berenguer RM, Bravet Rodríguez A, Reyes Fonseca AL, Salso Morell RA. Algunos factores de riesgo asociados a la estomatitis subprótesis. Revista Médica. Granma. 2019; 23(2).

Romero Junquera CR, García Rodríguez B, Genicio Ortega S,(2015). Estado prostodóncico y salud bucal en pacientes que solicitaron reparaciones protésicas. CCM 19(1). http://scielo.sld.cu/scielo.php

Ugarte M L, Azúcar López j, Cardoso I, Díaz Gómez S M, (2014). Influencia de la rehabilitación protésica en la calidad de vida de los pacientes. Rev Hum Med ; 14(3).http://scielo.sld.cu/scielo.php?pid=S172781202014000300004\&script=sci _arttext

\section{LCiencia}




\section{PARA CITAR EL ARTÍCULO INDEXADO.}

Espasandín González, S., González Díaz, Y., Reyes Suárez, V. O., \& González Casañas, B. Y. (2021). Agresiones protésicas a la mucosa bucal en pacientes geriátricos rehabilitados con prótesis estomatológicas removibles. Anatomía Digital, 4(4), 79-96. https://doi.org/10.33262/anatomiadigital.v4i4.1900

\section{Ligital}

El artículo que se publica es de exclusiva responsabilidad de los autores y no necesariamente reflejan el pensamiento de la Revista Anatomía Digital.

El artículo queda en propiedad de la revista y, por tanto, su publicación parcial y/o total en otro medio tiene que ser autorizado por el director de la Revista Anatomía Digital.
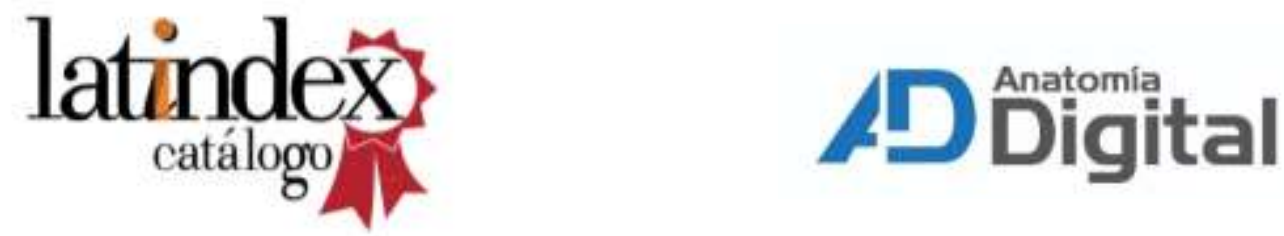